\title{
Atomic Opacities
}

\section{J. Seaton}

Department of Physics and Astronomy, University College London, Gower St., London WC1E 6BT, UK

\section{Introduction}

Let $I_{\nu}(r, \theta)$ be the intensity of radiation of frequency $\nu$, at a distance $r$ from the centre of a star and in a direction making an angle $\theta$ to the outward normal. In a plane-parallel approximation the equation of radiative transfer is

$$
\cos (\theta) \frac{\mathrm{d} I_{\nu}}{\mathrm{d} r}=-\kappa_{\nu} I_{\nu}+j_{\nu}
$$

where $\kappa_{\nu}$ is the opacity and $j_{\nu}$ the emissivity. The net outward flux of radiant energy is

$$
F_{\nu}(r)=\int_{-1}^{+1} I_{\nu}(r, \theta) \cos (\theta) \mathrm{d} \cos (\theta)
$$

and the flux integrated over all frequencies is

$$
F(r)=\int_{0}^{\infty} F_{\nu}(r) \mathrm{d} \nu .
$$

The source function is $S_{\nu}=j_{\nu} / \kappa_{\nu}$ and the optical depth is

$$
\tau_{\nu}(r)=\int_{r}^{\infty} \kappa_{\nu} \mathrm{d} r .
$$

In thermodynamic equilibrium at temperature $T, S_{\nu}=B_{\nu}(T)$ where

$$
B_{\nu}(T)=\left(2 h \nu 3 / c^{2}\right) /[\exp (h \nu / k T)-1] .
$$

The relation $S_{\nu}=B_{\nu}(T)$ can often be used even when one does not have full thermodynamic equilibrium; such situations are referred to as LTE (local thermodynamic equilibrium - see Johnson, this volume).

The expression for the opacity is

$$
\kappa_{\nu}=\sum_{i} N(i) \sigma_{\nu}(i)
$$


where $N(i)$ is the number-density of a species of type $i$ and $\sigma_{\nu}(i)$ is a cross section for absorption or scattering of radiation. In all opacity work there are two main physical problems to be tackled: obtain the number-densities $N(i)$ and the cross-sections $\sigma_{\nu}(i)$ used in Eq. (6).

For the astronomy work there are three cases to be considered:

1. NLTE, non-LTE. For the case of non-LTE one must take acount of all relevant radiative and collisional processes in order to calculate $N(i)$. The source-function $S_{\nu}$ depends on $I_{\nu}$, and hence on the solution of the equation of radiative transfer. NLTE effects are important for many problems concerned with the atmospheres of hot stars.

2. LTE. This is an adequate approximation for many stars. The level populations $N(i)$ are calculated using thermodynamic formulae. One may require large amounts of atomic and molecular data for radiative processes, but similar data for collisional processes are not required. The opacity $\kappa_{\nu}$ depends only on chemical composition and on the local variables of temperature $T$ and mass-density $\rho$.

3. Large optical depths, $\tau_{\nu} \gg 1$. For these cases LTE can be assumed. The transfer equation can be solved in the diffusion approximation (see, for example, Mihalas 1978) giving for the monochromatic radiative flux

$$
F_{\nu}=-\frac{4 \pi}{3} \frac{1}{\kappa_{\nu}} \frac{\mathrm{d} B_{\nu}}{\mathrm{d} r}
$$

The net flux is

$$
F=-\frac{4 \pi}{3} \frac{1}{\kappa_{R}} \frac{\mathrm{d} B}{d T} \frac{\mathrm{d} T}{d r}
$$

where $B(T)=\int B_{\nu}(T) \mathrm{d} \nu$ and where $\kappa_{R}$ is the Rosseland mean opacity defined by

$$
\frac{1}{\kappa_{R}} \frac{\mathrm{d} B}{\mathrm{~d} T}=\int_{0}^{\infty} \frac{1}{\kappa_{\nu}} \frac{\mathrm{d} B_{\nu}}{\mathrm{d} T} \mathrm{~d} \nu .
$$

In the present review I will be mainly concerned with the calculation of Rosseland mean opacities. They are of crucial importance for all work on the interiors of the stars. They are also important for work on stellar atmospheres, since the lower boundary conditions for an atmosphere are usually taken to be such as to match results obtained using the diffusion approximation.

\section{Equations of State - EOS}

Leaving aside the NLTE case, the calculation of level populations $N(i)$ is a problem of thermodynamics. For the molecular work that may be a matter of considerable intricacy but does not (so far as I am aware) present any great difficulties of principle. Such difficulties do arise when one considers the higher temperatures of stellar interiors. Let $N(j)$ be the number density for a given 
chemical element in ionisation stage $j$ and let $N(i, j)$ be the number in level $i$, $N(j)=\sum_{i} N(i, j)$. The familiar Boltzmann equation is

$$
N(i, j)=N(j) g(i, j) \exp [-E(i, j) / k T] / Q(j)
$$

where $g(i, j)$ is the statistical weight, $E(i, j)$ the excitation energy of the level and $Q(j)$ the partition function,

$$
Q(j)=\sum_{i} g(i, j) \exp [-E(i, j) / k T]
$$

The sum in Eq. (11) is divergent! Consider the case of the hydrogen atom and put $i=n$, the principal quantum number. As $n$ increases the energies $E(n)$ tend to a constant value (the ionisation limit) but $g(n)=2 n^{2}$ so that the integral diverges like $n^{2}$. The origin of this difficulty is, of course, that the highly excited states have very large radii, eventually becoming larger than the mean inter-particle separations in the plasma. In order to overcome the difficulty it is necessary to consider how the atomic states are modified by interactions with the plasma environment. It is not my purpose here to enter into further details about how that can be done: two references to recent work are Rogers (1991) and Hummer \& Mihalas (1988).

In principle, of course, the same difficulties do arise in the molecular work but they are of little practical importance since at lower temperatures the exponential factor in Eq. (11) becomes very small long before the divergence of the partition function starts to be a problem.

\section{Recent atomic opacity calculations}

For the calculation of atomic opacities one considers transitions of the following types: free-free (inverse bremsstrahlung); bound-free (photo-ionisation and photo-detachment); bound-bound (spectrum lines); and scattering (mainly by free electrons, also Rayleigh scattering at lower temperatures).

For many years astronomers have used Rosseland mean opacities from the Los Alamos Opacity Library, which I will refer to as LAOL (for references see Hübner (1985) and Weiss et al. (1990)). More recent calculations have been made by two independent teams: OPAL (Rogers \& Iglesias (1992), and Iglesias et al. (1992) - OPAL is the name of their computer code): and OP (Seaton et al. (1993) - the Opacity Project).

OPAL and OP use very different approaches to the EOS problems. OP uses the "chemical picture" in which atoms (and atomic ions) are assumed to exist but to be perturbed by the plasma environment. The OP work is restricted to stellar envelopes, that is to say to regions in which one need not take account of plasma perturbations in the calculation of the cross sections $\sigma_{i}(\nu)$. OPAL uses the "physical picture" and includes allowance for plasma perturbations in calculating the cross sections. OPAL go to deeper layers than those considered by OP. 
The two projects also have different approaches to the calculation of atomic radiative data. OPAL uses parametric model potentials with parameters adjusted to give good agreement for energy levels of free atoms. All cross-section data are calculated on-line as required and are not archived (that allows for the possibility of the cross sections being dependent on plasma conditions). The OP work, on the other hand, is based on very extensive $a b$ initio calculations for free atoms. Quite a large team has been involved (see Berrington et al. (1987) and Seaton et al. (1992)). Most of the work is done using elaborate R-matrix methods. Some supplementary calculations for large number of iron lines are also made using configuration-interaction codes. OP atomic data are archived in a database system TOPbase (see Cunto \& Mendoza (1992), and Cunto et al. (1993)).

Agreement between OPAL and OP results is generally satisfactory. For temperatures in the region of $T \simeq 2 \times 10^{5}$ the new opacities are larger than those from LAOL by factors as large as 3 or more. That has important consequences for theories of stellar pulsations. The enhancements are due to much more detailed treatments of atomic physics, in OPAL and OP compared with LAOL. Such detailed treatments are important for all of the "metals" (all elements other than $\mathrm{H}$ and $\mathrm{He}$ ), and are particularly important for ions of iron. For $T \simeq 2 \times 10^{5}$ the main ionisation stages for iron are Fe XV to Fe IX with ground-configurations $3 \mathrm{~s}^{2}$ to $3 \mathrm{~s}^{2} 3 \mathrm{p}^{6}$. Those ions have large number of spectral terms belonging to configurations of the type $3 \mathrm{~s}^{x} 3 \mathrm{p}^{y} 3 \mathrm{~d}^{z}$. Transitions between such terms, and excitations from them, give very large numbers of spectrum lines - even more when finestructure splittings are taken into account. Molecular spectroscopists who think that their spectra are complicated because there are many lines should take a look at the complexity of the iron spectra in the vicinity of $2 \times 10^{5} \mathrm{~K}$, as shown on Figure 22a of Rogers \& Iglesias (1992) or Figure 10 of Seaton et al. (1993).

\section{Fitting and smoothing}

The Rosseland mean opacity $\kappa_{R}$ depends on temperature $T$, density $\rho$ and chemical composition. Work on stellar structures requires, for each specified composition, a knowledge of $\kappa_{R}(T, \rho)$ and of the first two derivative,

$$
\left.\frac{\partial \kappa_{R}}{\partial T}\right|_{\rho} \text { and }\left.\frac{\partial \kappa_{R}}{\partial \rho}\right|_{T} .
$$

Higher derivatives may also be required. The calculations of $\kappa_{R}(T, \rho)$ are made with intervals in $T$ and $\rho$ such that quadratic or cubic interpolations can be used. There is, however, one practical difficulty which arises.

In order to resolve all structures in the monochromatic opacities $\kappa_{\nu}$ it would be necessary to use $10^{6}$ or even $10^{7}$ frequency points in evaluating the Rosseland integral, which would be prohibitively expensive. In practice the calculations are made with some $10^{4}$ frequency points. The technique is that of opacity sampling, which is also used in much of the molecular work. It has been checked that the use of sampling does not lead to errors larger than two or three per cent in $\kappa_{R}$ which is not in itself of much importance, since for other reasons $\kappa_{R}$ will not 
be accurate to better than a few per cent. The practical difficulty is that the errors can change in a more-or-less random fashion as one passes from one $(T, \rho)$ grid-point to the next, and can therefore lead to quite large errors in calculated derivatives, which take on an unpleasant "wiggly" appearance.

I have recently shown that the problem can be overcome using a modest amount of smoothing (Seaton (1993)). I use indices $i$ and $j$ for grid-points in $T$ and $\rho$ and in the vicinity of a grid-point $(i, j)$ I consider the 25 neighbouring points $(i+n, j+m), n=-2$ to +2 and $m=-2$ to +2 ; and make a leastsquares fit to a bi-cubic involving 16 coefficients. The value of $\kappa_{R}$ at the point $(i, j)$ is then replaced by the value obtained from the fit. It is found that the differences between the original and the smoothed data are no larger than the errors which can be expected to arise from the use of opacity sampling; and using the smoothed data the calulated derivatives have a much more reasonable. and pleasing appearance.

\section{Access to atomic opacity data}

\subsection{OPAL opacities}

OPAL opacity tables are available from the Lawrence Livermore National Laboratory via ftp dplasma.llnl.gov, user anonymous and cd /pub/opacity.

\subsection{TOPbase}

The OP atomic data database, TOPbase, is installed at the Centre des Donées Astronomiques de Strasbourg (CDS). Using ftp 130.79.128.5 with anonymous login and cd /pub/topbase one can obtain the TOPbase User Manual userman.tex as a $\mathrm{IAT}_{\mathrm{E}} \mathrm{X}$ file. To access the database itself one uses telnet 130.79.128.5, -account : topbase, and -password: Seaton+

\subsection{OP opacities}

OP opacity data for any required chemical mixture, and the fitting-and-smoothing codes (OPALFIT and OPFIT), can be obtained on sending e-MAIL messages to one of the following:-

- Dr. A.E. Lynas-Gray, Astrophysics, Keble Road, Oxford, OX1 3RH, UK. Telephone: +44-865-73363.

Electronic mail (INTERNET): aelg@oxds02.astro.ox.ac.uk

- Dr. Anil K. Pradhan, Department of Astronomy, 174 W. 18th St., The Ohio State University, Columbus, Ohio 43210-1106, USA.

Telephone: +614-292-5850.

Electronic mail (INTERNET): pradhan@seaton.mps.ohio-state.edu 


\section{The marriage of atomic opacities with molecular opacities}

Throughout most of a stellar interior the radiative opacity is determined almost entirely by atomic processes, the temperatures being too high for molecules to exist with appreciable abundances. That is no longer true for the outer layers of cooler stars. Those who calculate Rosseland mean opacities are constantly faced with the complaint that their tables do not go to sufficiently low temperatures. That position can be rectified only when molecular contributions are included.

Those who calculate molecular opacities have given particular attention to the outer layers of cool stars, that is to say regions in which the opacity is largely dominated by molecular contributions. For the calculations of Rosseland mean opacities there is an urgent need to bridge the gap between regions in which molecular contributions are dominant and those in which the atomic contributions are dominant. It is required to calculate monochromatic opacities, $\kappa_{\nu}$, with inclusion of all contributions, before evaluation of the Rosseland integral Eq. (9). I hope that the present book will provide a stimulus for such work to be done.

Acknowledgements. I thank Carlos Iglesias and Forrest Rogers for permission to give details concerning $f t p$ access to their opacity data.

\section{References}

Berrington K.A., Burke P.G., Butler K., Seaton M.J., Storey P.J., Taylor K.T., Yu Yan, 1987, J. Phys. B., 20,6363

Cunto W., Mendoza C., 1992, Rev. Mex. Astron. Astrofis., 23, 107

Cunto W., Mendoza C., Ochsenbein F., Zeippen C.J., 1993, Astron. Astrophys., in press

Hummer D.G., Mihalas D., 1988, Astrophys. J., 331, 794

Hübner W.F., 1985, In: P.Sturrock, T.Holzer, D.Mihalas (eds.), Physics of the Sun, (Dord recht: Reidel), p.33

Iglesias C.A., Rogers F.J., Wilson B.G., 1992, Astrophys. J., 397, 717

Mihalas D., 1978, Stellar Atmospheres, 2nd ed., (San Francisco: Freeman)

Rogers F.J., 1992, In: S. Eliezer, R.A. Ricci (eds.), High Pressure Equations of State: Theory and Applications, (New York: North Holland), p.77

Rogers F.J., Iglesias C.A., 1992, Astrophys. J. Supp., 79, 507

Seaton M.J., Zeippen C.J., Tully J.A., Pradhan A.K., Mendoza C., Hibbert A., Berrington C.A., 1992, Rev. Mex. Astron. Astrofis., 23, 19

Seaton M.J., Yu Yan, Mihalas D., Pradhan A.K., 1993, Mon. Not. R. Astr. Soc., in press

Seaton M.J., 1993, Mon. Not. R. Astr. Soc., submitted

Weiss A., Keady J.J., Magee N.H., 1990, Atomic Data Nucl. Data Tables, 45, 209 